\title{
The Effect and Acceptability of Increased Whole Apple Consumption, Apple Extract and Freeze-Dried Apple Pieces on Biomarkers of CVD Risk: The APPS Pilot Study
}

\author{
Sarah-Louise Henry ${ }^{1}$, Sharon Fulton ${ }^{1}$, Lesley Hamill ${ }^{1}$, Kirsti Tiihonen ${ }^{2}$, Oliver Hasselwander ${ }^{3}$, \\ Amy C Wotherspoon ${ }^{1}$, Michelle C McKinley ${ }^{1}$, Jayne V Woodside ${ }^{1, *}$ \\ ${ }^{1}$ Centre for Public Health, Queen's University Belfast \\ ${ }^{2}$ DuPont, Nutrition and Health, Kantvik, Finland \\ ${ }^{3}$ DuPont, Nutrition and Health, Reigate, UK \\ *Corresponding author: j.woodside@qub.ac.uk
}

\begin{abstract}
An increased intake of fruit and vegetables has been associated with decreased risk of cardiovascular disease (CVD). However, it is currently unclear as to whether particular fruits and vegetables convey more benefit than others, and if the form in which a particular fruit or vegetable is delivered, for example, depending on polyphenol content or the degree of processing, affects potential health benefits. The aim of this study was to conduct a pilot randomised placebo-controlled trial (RCT) with the ultimate aim of assessing if different apple products differentially affect CVD risk factors. The RCT was conducted in those at elevated risk of CVD. Participants were randomised to receive high polyphenol apples, low polyphenol apples, capsules containing apple extract, dried apple pieces or placebo capsules to take for four weeks. A range of risk factors and biomarkers associated with CVD were measured before and after the intervention period. A total of 55 participants were randomised and completed the study. Change in hip circumference was significantly different across the groups after 4 weeks $(P=0.02)$ Change in fibre intake was also statistically significant between groups, with those consuming high polyphenol apples having a higher intake than those on the apple extract $(P=0.01)$. There was a significant within-group change in fasting oxidised LDL in the apple extract group $(P=0.008)$. A significant difference in change in volume-corrected epicatechin was observed $(P=0.03)$, with those on apple extract having higher levels than those on placebo capsule $(P=0.002)$ or low polyphenol apples $(P=0.01)$ Overall, interventions were generally acceptable to participants. Apple products appeared to have a positive effect on some risk factors for CVD in this pilot study, which indicated a definitive RCT is feasible. Further adequately powered studies need to be conducted to definitively test these findings.
\end{abstract}

Keywords: apples, cardiovascular disease, biomarkers, acceptability, feasibility, RCT, epicatechin

Cite This Article: Sarah-Louise Henry, Sharon Fulton, Lesley Hamill, Kirsti Tiihonen, Oliver Hasselwander, Amy C Wotherspoon, Michelle C McKinley, and Jayne V Woodside, "The Effect and Acceptability of Increased Whole Apple Consumption, Apple Extract and Freeze-Dried Apple Pieces on Biomarkers of CVD Risk: The APPS Pilot Study." Journal of Food and Nutrition Research, vol. 5, no. 12 (2017): 882-893. doi: 10.12691/jfnr-5-12-2.

\section{Introduction}

Abundant observational evidence suggests an association between increased consumption of fruit and vegetables and decreased risk of major diseases, such CVD $[1,2]$. However, to date, few intervention studies have been carried out to support this observational evidence, although an effect of increased fruit and vegetable consumption has been shown, for example, on microvascular function [3] and blood pressure [4].

Whilst increased consumption of fruit and vegetables is generally accepted as being beneficial to health, intakes remain below the 5 portions per day recommendation $[5,6]$.
Barriers to increasing intakes of fruit and vegetables include cost, liking, poor nutritional knowledge, and accessibility $[7,8,9,10]$. The low intake of fruit and vegetables combined with the presence of these reported barriers to change demonstrates the need for new approaches to increase fruit and vegetable consumption within the general population. Low consumption of fruit and vegetables has meant that products which attempt to mimic the health benefits of a diet rich in fruit and vegetables (such as extracts and dried products) have been developed. However, it is not known if similar health benefits can be gained from consuming such products or whether the health benefits are specific to the consumption of the whole food [11].

It has also yet to be established whether certain types of fruit and/or vegetables are associated with greater health 
benefits and the likely mechanisms through which these foods act. For example, in a recent review by Chong et al. [12], fruit polyphenols showed some evidence of being effective in reducing CVD risk factors in short-term studies. Apples are an example of a polyphenol-rich food, and are one of the most commonly consumed fruits in Europe, and, indeed, the UK $[13,14,15]$. They possess the second highest antioxidant activity of all fruits [16], which has been attributed to their main antioxidant, epicatechin.

The aim of this study was to conduct a pilot randomised controlled trial (RCT) to assess the feasibility of testing whether different apple products can modify risk factors associated with CVD.

\section{Materials and Methods}

The APPS (Apple/Apple Products) study was a pilot 4-week randomised placebo-controlled trial with a double blind component. Participants at increased risk of CVD were recruited from the general population through poster advertisement, staff intranet postings within the university, as well as advertisement in a local community group of retired adults. Individuals who expressed interest in participating in the study were sent participant information sheets and a telephone number and email address for the study researcher was provided if they had questions regarding the study. Participants were eligible if they met one of the following criteria: aged $>50$ years, current smoker, elevated systolic blood pressure $(120-139 \mathrm{mmHg})$ or diastolic blood pressure $(80-89 \mathrm{mmHg})$, total cholesterol $>5.2 \mathrm{mmol} / \mathrm{L}$ or HDL cholesterol $<1.03 \mathrm{mmol} / \mathrm{L}$ or BMI $>25 \mathrm{~kg} / \mathrm{m}^{2}$. Exclusion criteria were as follows: diagnosis of diabetes mellitus, acute coronary syndrome or transient ischaemic attack within past 3 months, special dietary requirements or food sensitivities or on vegetarian/vegan diet through choice, on oral anticoagulation therapy, BMI $>35 \mathrm{~kg} / \mathrm{m}^{2}$, excessive alcohol consumption ( $>28 \mathrm{U} /$ week for men or $>21 \mathrm{U} /$ week for women), pregnant/lactating taking antioxidant supplements or medial conditions/dietary restrictions that would substantially limit ability to complete study requirements. The study was approved by the Research Ethics Committee of the School of Medicine Dentistry and Biomedical Sciences, Queen's University Belfast. All participants gave informed written consent.

\subsection{Randomisation}

Participants were randomised to one of five groups at week 0 (baseline visit), using a block design (with blocks of $n=10$ ) and computer-generated numbers. Participants were randomised to consume one of the following, for 4 weeks: an apple extract (encapsulated; two consumed/day), two high polyphenol apples/day, two low polyphenol apples/day, a low apple diet $(<1$ portion/day of apples or apple products), or $9 \mathrm{~g}$ of freeze dried apple pieces/day. In addition, all groups, with exception of the group receiving the apple extract capsule, also consumed two placebo capsules, meaning that this component of the study was double blinded. All groups, with the exception of the low polyphenol apple and high polyphenol apple groups, were advised to follow a low apple diet ( $<1$ portion/day). The apple varieties from which the products were made were
UK-grown and were particularly rich in epicatechin. The high polyphenol apple, apple extract and freeze dried apple pieces groups were standardised for epicatechin intake of approximately $80 \mathrm{mg} /$ day (data supplied by manufacturer of food products/apple producer; Coressence, Bristol, UK). Participants were advised to keep intake of all other polyphenol-rich foods (i.e. tea, coffee, wine, other fruit and vegetables) constant and were told not to start taking any vitamin/mineral supplements whilst enrolled on the study. Apples were delivered by the researchers on a weekly basis to participants in the apple groups, whilst all apple products (i.e. dried apple pieces, apple extract) and placebo capsules were given to the participants at the baseline visit.

To encourage compliance with the study, participants were given written advice at the baseline visit on how to incorporate the apples and apple products into their diet, as well as advice on storage. Participants were informed not to cook the apples to preserve their epicatechin content. Information booklets also contained advice on how to collect urine samples, as well as reminders not to change diet or lifestyle habits and to fast before the study visits. All participants were given a chart to log consumption of study products. Participants were telephoned weekly to ensure they were consuming study products and also to monitor body weight. A payment of $£ 30$ was given to participants after successful completion of the study, which covered travel to and from study visits. Compliance was assessed using self-reported dietary intake collected using 4-day diet diaries; these were completed at baseline (habitual diet) and week 4 (incorporation of apples).

\subsection{Procedures}

Two urine samples were collected prior to each study visit (description below). During study visits, a spot urine sample, fasted and 2-hour post prandial blood samples, anthropometric data (weight, height and waist circumference) were collected and blood pressure was measured

\subsubsection{Anthropometric Data}

Height was measured using a Leicester portable height measure. Participants removed their footwear and stood upright with their feet together and shoulder blades touching the vertical backboard. The moveable headboard was lowered to the crown of the head, which was in the Frankfort horizontal plane. Measurements were recorded to the nearest $0.1 \mathrm{~cm}$. Weight was measured using calibrated digital weight scales (Seca 876, SECA, Birmingham, UK). Participants removed any heavy articles of clothing and shoes. Measurements were recorded to the nearest $0.1 \mathrm{~kg}$. Waist and hip circumference were assessed using a flexible measuring tape. Participants were asked to remove any outer garments (i.e. coats) and stand upright, with their arms by their sides, feet together while relaxing their abdominal muscles. Waist circumference was measured at the midpoint between the ribcage and iliac crest, and where this point could not be located (i.e. in cases of extreme obesity), the widest point was measured. Hip circumference was then measured, with the participant being asked to remain in the standing position and the widest point around the buttocks measured to the 
nearest $0.1 \mathrm{~cm}$. Waist-hip ratio was calculated as waist circumference $(\mathrm{cm}) /$ hip circumference $(\mathrm{cm})$.

\subsubsection{Urine Sampling}

Participants collected an overnight urine sample, which was to be collected between after their evening meal until midnight prior to each visit.

\subsubsection{Blood Sampling}

Fasted blood samples and a 2-hour postprandial blood samples were collected at baseline and the end of the study. For postprandial blood samples, participants received a standardised breakfast (cornflakes with semi-skimmed milk) along with their study product for that day prior to sampling. Serum samples were placed in the dark at room temperature, whereas plasma samples were inverted immediately after being drawn and kept at $4^{\circ} \mathrm{C}$ for 30 minutes before centrifugation of both samples at $3000 \mathrm{rpm}$ for 15 minutes at $4^{\circ} \mathrm{C}$. All samples were then stored at $-80^{\circ} \mathrm{C}$ until analysis.

\subsubsection{Blood Pressure Measurement}

Blood pressure was measured using an Omron M5-1 automatic blood pressure monitor (Omron Healthcare, The Netherlands). Three consecutive readings were taken and an average of the $2^{\text {nd }}$ and $3^{\text {rd }}$ readings was recorded

\subsubsection{Dietary Assessment}

Dietary intake was assessed using a 4-day estimated diet diary. Participants were instructed to complete the diet diary as per normal consumption, over a consecutive 4-day period before attending for the assessment visits. Completed diet diaries were analysed using the dietary analysis programme WISPv3.0 (Weighed Intake Software Program; Tinuviel Software, Warrington, U.K.).

\subsubsection{Biochemical Analysis}

Oxidised LDL was determined in both fasting and postprandial plasma samples by ELISA (Mercodia, Uppsala, Sweden). ICAM-1 was determined in fasting and postprandial serum samples by ELISA (Quantikine, R\&D systems Inc., Minneapolis, USA). VCAM-1 was determined in fasting and postprandial samples by ELISA (Quantikine, R\&D systems Inc., Minneapolis, USA). E-Selectin was determined in fasting and postprandial samples by ELISA (Quantikine, R\&D systems Inc., Minneapolis, USA). CRP was determined in fasting serum using an I-Lab 600 automated analyser. Carotenoids (lutein, zeaxanthin, $\beta$ - cryptoxanthin, $\alpha$-carotene, $\beta$-carotene) were extracted from serum using the method of Craft et al. [17]. Vitamin $\mathrm{C}$ (ascorbic acid) was determined by fluorometric method [18] in plasma treated with 5\% metaphosphoric acid. Epicatechin content was determined in serum samples and non-fasting urine samples (e.g. collected from participant after evening meal until midnight) by HPLC-MS/MS (Supplementary material). This in-house method is based on a number of published techniques and measures a range of polyphenols. The enzymatic extraction and chromatographic-mass spectrophotometer conditions are based on those described by Ito et al. (2005). Urine samples were dried based on the method of Nielsen et al [19]. Sample preparation (except for the solid phase extraction step after hydrolysis), was similar to that described by Rein et al. [20] and Richelle et al. [21].

\subsubsection{Urinary Creatinine Standardisation of Epicatechin Concentrations}

Creatinine was measured by a colorimetric method using a commercially available kit (Randox Laboratories Ltd, Crumlin, UK) modified for the Ilab 600 (Instrumentation Laboratory, Warrington, UK). These were run in several batches (in consecutive numerical order, containing baseline and week 4 samples simultaneously for each participant). The mean $\mathrm{CV}$ for intra-batch and inter-batch urine controls were both $2.3 \%$. Results are presented as nmol epicatechin per $\mathrm{mg}$ creatinine ( $\mathrm{nmol} / \mathrm{mg}$ creatinine).

\subsubsection{Urinary Volume Standardisation of Epicatechin Concentrations}

Volume of urine samples was measured in the laboratory using a measuring cylinder (to the nearest $10 \mathrm{ml}$ ). Epicatechin data presented in $\mathrm{nmol} / \mathrm{mL}$ were multiplied by volume and results are presented as nmol epicatechin per sample (nmol/sample).

\subsubsection{Study Questionnaires}

Participants were asked to complete three questionnaires: a medical history and lifestyle questionnaire, a levels of liking questionnaire and a tolerability questionnaire. Study questionnaires were in electronic format, using the online survey software Qualtrics. Participants completed the questionnaires whilst attending study visits (weeks 0 and 4). In addition to this, the levels of liking questionnaire was emailed to participants at week 2 of the intervention.

\subsubsection{Medical History \& Lifestyle Questionnaire}

Data were collected on each participant's medical history and lifestyle habits via questionnaire at each visit. The questionnaire assessed vitamin, mineral and food supplement use, smoking and drinking habits, years spent in full-time education, occupational status and current medication use.

\subsubsection{Levels of Liking Questionnaire}

Liking of 70 different foods was assessed based on the structure of the EPIC food frequency questionnaire (FFQ) [22]. This FFQ had food frequencies replaced with a 9-point hedonic scale (like extremely, like very much, like moderately, like slightly, neither like nor dislike, dislike slightly, dislike moderately, dislike very much, dislike extremely), which was then used to assess how much or how little participants liked or disliked the foods listed. An option was also given for items which the participant had never tried. The foods were broken down into sections: sweets and snacks (e.g. cakes, biscuits, chocolate), fruit (e.g. apples, pears, bananas), vegetables (e.g. carrots, broccoli, brussel sprouts), bread and savoury biscuits (e.g. white bread and rolls, cheese biscuits) and cereals (e.g. breakfast cereals, porridge). The participants were asked about each food individually and could only give one answer about how much or how little they liked each food. The scale was scored from $1=$ dislike extremely to 9=likely extremely, while an option was also given if participants had never tried the food, and this was given a 
score of 0 . Total fruit and vegetable scores were calculated by summing together the individual fruit/vegetable scores for each participant. Apple liking scores were also considered separately.

\subsubsection{Tolerability Questionnaire}

At the end of the intervention, participants' tolerability of study products they had consumed was assessed. Questions surrounded any side effects, ease of consumption, acceptability, willingness to continue taking study product post-intervention and willingness to purchase study product. The questionnaire was amended from one used in a similar study [23]. Questionnaires were obtained from the study authors.

\subsection{Statistical Analysis}

Statistical analysis was performed using SPSS v21.0 (IBM Corp., Armonk, NY). Quantitative analyses were conducted, but, as this was a pilot study, the main aim was to explore feasibility, to develop hypotheses and to generate data to allow future power calculations. Baseline values and change values (calculated as week 4 - week 0 ) were compared between groups using one way analysis of variance (ANOVA) with Student Newman Keuls (SNK) post-hoc tests, while comparisons between low and high epicatechin containing groups, and between the placebo capsule and apple extract groups (double-blinded component) were made using student independent samples t-tests. Associations between continuous variables were examined using Pearson correlation coefficients. $\mathrm{P}$ values of $<0.05$ were considered significant.

\section{Results}

\subsection{Summary of APPS Study Recruitment}

Figure 1 shows flow of participants through the study. Screening and recruitment for the APPS study took place between April 2012 and May 2013. In total, 86 individuals were screened, of which $30(34.8 \%)$ were not eligible to take part due to: blood pressure readings being greater than specified (i.e. Systolic BP $>140 \mathrm{mmHg}$ and/or Diastolic BP $>90 \mathrm{mmHg})(11.6 \%, \mathrm{n}=10)$; BMI being $>35$ $\mathrm{kg} / \mathrm{m}^{2}(3.8 \% \mathrm{n}=3)$; individuals not having a CVD risk factor $(5.8 \%, \mathrm{n}=5)$; individuals already taking part in another intervention study $(2.3 \%, \mathrm{n}=2)$; individuals taking regular vitamin $\mathrm{C}$ supplements $(3.8 \%, \mathrm{n}=3)$; individuals who changed their mind about taking part in the study during screening $(2.3 \%, \mathrm{n}=2)$; those currently reporting problematic angina $(2.3 \%, \mathrm{n}=2)$; individuals who were vegetarian $(1.2 \%, \mathrm{n}=1)$ and those unable to find time to take part $(1.2 \%, \mathrm{n}=1)$. One further person was eligible but dropped out at the beginning of the study due to personal reasons. Therefore, 55 participants were recruited and commenced the 4 week study. All 55 participants completed all study requirements.

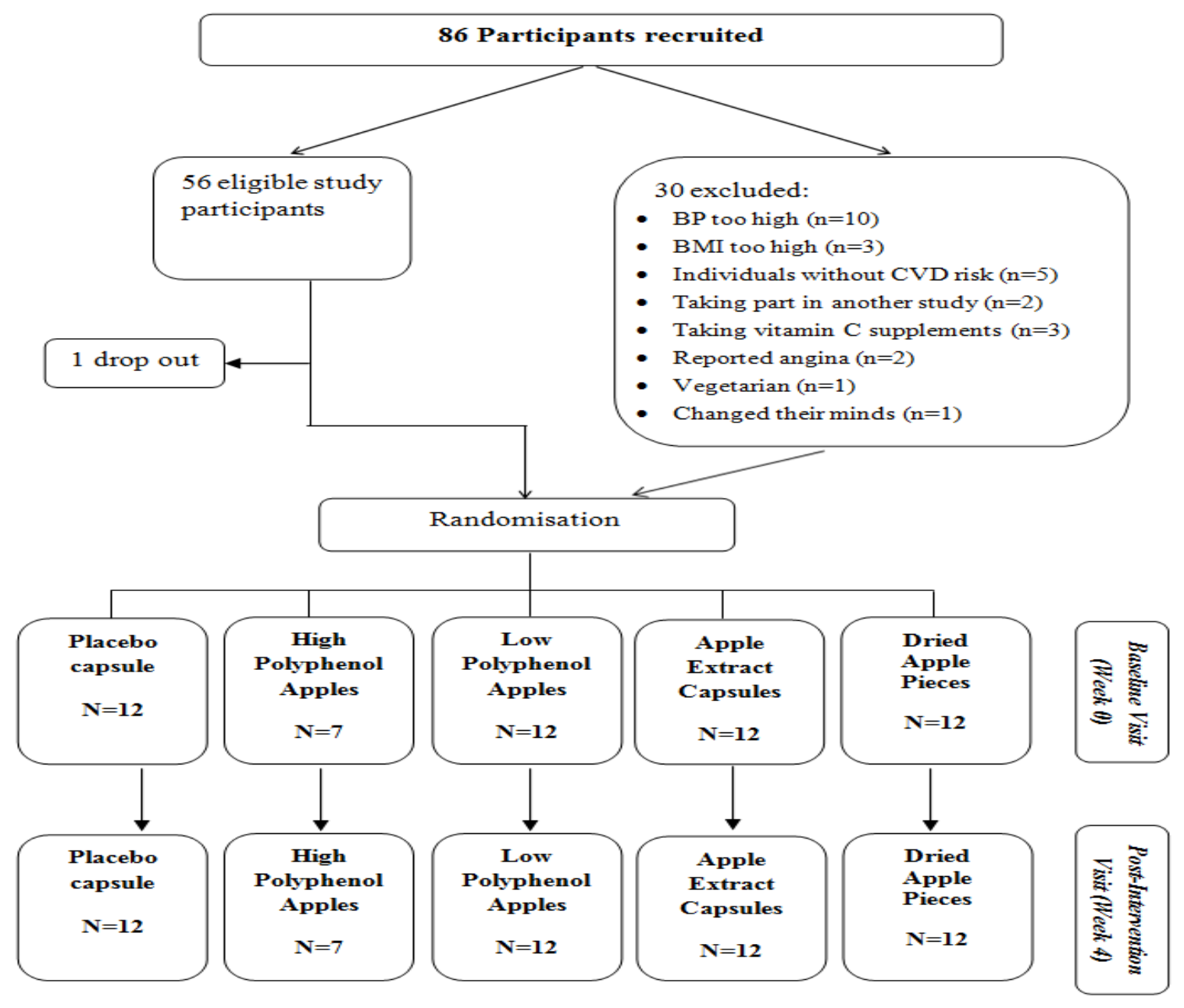

Figure 1. Figure illustrating APPS study numbers 


\subsection{Baseline Characteristics}

Baseline characteristics for study participants are shown in Table 1 . The study was made up of $53 \%$ males and $47 \%$ females, with a mean age of 40.6 years. Three of the four intervention groups (apple extract, low polyphenol apple, and apple pieces) and the placebo control group had 12 participants, whilst the high polyphenol group had 7 participants (only 7 participants could be randomised to this group due to availability of study apples). In total, $18 \%$ of participants took micronutrient or other supplements whilst participating in the study (however, no participants were taking supplements which would have interfered with study outcomes such as nutritional biomarkers measured), 20\% were current smokers (with 9\% being past smokers), $47 \%$ were alcohol consumers and $35 \%$ were currently using medication. No significant differences between baseline characteristics were observed.

Table 1. Baseline characteristics of APPS study participants, according to intervention group

\begin{tabular}{|c|c|c|c|c|c|c|}
\hline & Total & $\begin{array}{c}\text { Placebo Capsule } \\
(N=12)\end{array}$ & $\begin{array}{c}\text { Apple Extract } \\
(N=12)\end{array}$ & $\begin{array}{c}\text { High Polyphenol Apple } \\
(N=7)\end{array}$ & $\begin{array}{c}\text { Low Polyphenol Apple } \\
(N=12)\end{array}$ & $\begin{array}{c}\text { Apple Pieces } \\
(N=12)\end{array}$ \\
\hline Females & $26(47.3)$ & $4(33.3)$ & $5(41.7)$ & $4(57.1)$ & $6(50)$ & $7(58.4)$ \\
\hline Age, years & $40.6(17.0)$ & $42.2(18.1)$ & $43.9(17.8)$ & $34.7(18.1)$ & $38.8(13.7)$ & $41.0(19.3)$ \\
\hline$B M I\left(\mathrm{~kg} / \mathrm{m}^{2}\right)$ & $26.5(4.1)$ & $25.9(3.0)$ & $26.2(3.5)$ & $27.2(4.2)$ & $25.5(4.3)$ & $27.9(5.4)$ \\
\hline Waist (cm) & $90.8(12.6)$ & $88.1(8.8)$ & $91.2(12.0)$ & $89.1(13.3)$ & $88.5(11.2)$ & $98.4(17.0)$ \\
\hline Hip (cm) & $104.5(6.2)$ & $104.4(5.4)$ & $103.5(3.4)$ & $105.8(6.9)$ & $102.3(5.7)$ & $107.1(8.6)$ \\
\hline Waist to hip ratio & $0.87(0.1)$ & $0.84(0.06)$ & $0.88(0.10)$ & $0.84(0.09)$ & $0.86(0.09)$ & $0.90(0.10)$ \\
\hline$S B P(m m H g)$ & $123.8(10.6)$ & $118.4(9.5)$ & $126.8(8.7)$ & $121.6(13.2)$ & $125.3(9.7)$ & $126.8(8.7)$ \\
\hline$D B P(m m H g)$ & $79.2(8.0)$ & $75.3(5.7)$ & $79.0(10.4)$ & $77.0(8.4)$ & $91.3(7.1)$ & $82.5(6.8)$ \\
\hline Vitamin Use & $10(18.2)$ & $3(25)$ & $3(33.3)$ & 0 & $1(8.3)$ & $2(16.7)$ \\
\hline Current Smoker & $11(20.0)$ & $4(33.3)$ & $2(16.7)$ & $1(14.3)$ & $4(33.3)$ & 0 \\
\hline Alcohol Consumers & $25(47.3)$ & $6(50)$ & $8(66.7)$ & $1(11.1)$ & $7(58.3)$ & $4(33.3)$ \\
\hline Using Medication & $19(34.5)$ & $3(25)$ & $6(50)$ & $1(14.3)$ & $5(41.7)$ & $4(33.3)$ \\
\hline
\end{tabular}

Data presented as mean (SD) or $\mathrm{n}(\%)$.

Table 2. Change in anthropometric measurements and blood pressure after 4-week intervention with apples and apple products, according to intervention group

\begin{tabular}{|c|c|c|c|c|c|}
\hline & & $N=$ & Week 0 & Week 4 & Change at week 4 \\
\hline \multirow{5}{*}{$\begin{array}{l}\text { Weight } \\
\text { (kg) }\end{array}$} & Placebo Capsule & 12 & $80.3(3.38)$ & $80.5(3.35)$ & $0.19(-0.58,0.96)$ \\
\hline & Apple Extract & 12 & $80.4(4.37)$ & $80.6(4.42)$ & $0.22(-0.31,0.74)$ \\
\hline & High Polyphenol Apple & 7 & $76.7(5.13)$ & $77.1(4.88)$ & $0.47(-0.22,1.16)$ \\
\hline & Apple Pieces & 12 & $82.2(6.03)$ & $82.7(6.17)$ & $0.77(-0.06,1.59)$ \\
\hline & Between group $\mathbf{P}$ Value & & 0.58 & 0.79 & 0.54 \\
\hline \multirow{5}{*}{$\begin{array}{l}B M I \\
\left(\mathrm{~kg} / \mathrm{m}^{2}\right)\end{array}$} & Placebo Capsule & 12 & $25.9(0.85)$ & $25.9(0.80)$ & $0.06(-0.19,0.30)$ \\
\hline & High Polyphenol Apple & 7 & $27.2(1.58)$ & $27.4(1.54)$ & $0.19(-0.05,0.42)$ \\
\hline & Low Polyphenol Apple & 12 & $25.5(1.23)$ & $25.6(1.23)$ & $0.05(-0.21,0.31)$ \\
\hline & Apple Pieces & 12 & $27.9(1.57)$ & $28.1(1.61)$ & $0.17(-0.00,0.33)$ \\
\hline & Between group P Value & & 0.63 & 0.58 & 0.83 \\
\hline \multirow{5}{*}{$\begin{array}{l}\text { Waist Circumference } \\
\text { (cm) }\end{array}$} & Placebo Capsule & 12 & $88.1(2.53)$ & $87.6(2.19)$ & $-0.47(-1.61,0.68)$ \\
\hline & Apple Extract & 12 & $91.2(3.46)$ & $91.3(3.51)$ & $0.13(-0.31,0.56)$ \\
\hline & High Polyphenol Apple & 7 & $89.1(5.03)$ & $89.0(4.95)$ & $-0.07(-0.63,0.49)$ \\
\hline & Low Polyphenol Apple & 12 & $88.5(3.22)$ & $88.5(3.18)$ & $0.04(-0.67,0.75)$ \\
\hline & Between group P Value & & 0.50 & 0.43 & 0.59 \\
\hline \multirow{6}{*}{$\begin{array}{l}\text { Hip Circumference } \\
(\mathrm{cm})\end{array}$} & Placebo Capsule & 12 & $104(1.55)$ & $104(1.49)$ & $-0.21(-1.00,0.59)$ \\
\hline & Apple Extract & 12 & $104(0.99)$ & $104(1.03)$ & $0.53(-0.04,1.09)$ \\
\hline & High Polyphenol Apple & 7 & $106(2.62)$ & $105(2.64)$ & $-1.11(-2.35,0.12)$ \\
\hline & Low Polyphenol Apple & 12 & $102(1.66)$ & $101(1.72)$ & $-0.92(-1.86,0.03)$ \\
\hline & Apple Pieces & 12 & $107(2.50)$ & $107(2.50)$ & $-0.35(-0.94,0.24)$ \\
\hline & Between group P Value & & 0.38 & 0.35 & 0.02 \\
\hline \multirow{6}{*}{$\begin{array}{l}\text { Systolic Blood } \\
\text { Pressure }(\mathrm{mmHg})\end{array}$} & Placebo Capsule & 12 & $118(2.75)$ & $117(2.51)$ & $-1.25(-5.50,3.00)$ \\
\hline & Apple Extract & 12 & $126(3.50)$ & $122(3.90)$ & $-4.50(-9.33,0.33)$ \\
\hline & High Polyphenol Apple & 7 & $152(4.97)$ & $122(7.52)$ & $0.14(-8.94,9.23)$ \\
\hline & Low Polyphenol Apple & 12 & $125(2.81)$ & $122(1.93)$ & $-3.25(-8.71,2.21)$ \\
\hline & Apple Pieces & 12 & $127(2.51)$ & $121(3.15)$ & $-5.33(-10.9,0.22)$ \\
\hline & Between group P Value & & 0.27 & 0.84 & 0.57 \\
\hline \multirow{4}{*}{$\begin{array}{l}\text { Diastolic Blood } \\
\text { Pressure (mmHg) }\end{array}$} & Placebo Capsule & 12 & $75.3(1.65)$ & $75.3(1.64)$ & $0.00(-3.44,3.44)$ \\
\hline & Apple Extract & 12 & $79.0(2.99)$ & $78.2(2.89)$ & $-0.83(-4.70,3.04)$ \\
\hline & Apple Pieces & 12 & $82.5(1.96)$ & $80.7(3.00)$ & $-1.83(-5.37,1.71)$ \\
\hline & Between group $P$ Value & & 0.17 & 0.54 & 0.91 \\
\hline
\end{tabular}

Absolute variables are presented as mean (SD), change at week 4 presented as mean $(95 \% \mathrm{CI})$. Baseline values and changes were compared between groups using one way ANOVA with SNK post-hoc tests. Presentation of mean change (95\% CI) allows examination of any statistically significant within group changes (i.e. any confidence intervals that do not cross 1.0). 


\subsection{Change in Anthropometric Measurements/Blood Pressure during the Intervention}

Data for anthropometric measurements is presented in Table 2. No significant differences in change in weight, BMI, waist circumference, systolic blood pressure and diastolic blood pressure over the course of the intervention were shown between groups, however, there was a significant difference between groups in change in hip circumference over the 4 -week intervention $(p=0.02)$, with those in the high polyphenol apple group having a significantly larger change than those in the apple extract group. No significant within group changes were identified over the course of the intervention.

\subsection{Change in Dietary Intake during the Intervention}

There were no significant differences between groups at week 0 for any of the macro- and micronutrient intakes analysed (data not shown). Differences between groups, in change at week 4 , were not statistically significant for the majority of macro- and micronutrients intakes with the exception of change at week-4 for dietary fibre intake between groups $(p=0.01)$, with those in the high polyphenol apple group having a significantly larger change in fibre intake than those in the apple extract group (data not shown).

\subsection{Biomarkers of CVD Risk}

Biomarkers that were measured as part of the study are presented in Table 3.

\subsubsection{Lipid Biomarkers}

There was no significant difference for change in lipids at week-4 between groups. Similarly, there was no between group difference for change in the primary endpoint, fasting oxidised LDL or post-prandial oxidised LDL. However, there was a significant within-group change in fasting oxidised LDL in the apple extract group $(\mathrm{p}=0.008)$.

\subsubsection{Inflammatory Biomarkers}

There was no significant difference for change in inflammatory biomarkers at week-4, although the difference in change at week- 4 in CRP did tend towards significance between groups $(\mathrm{p}=0.091)$.

\subsubsection{Vitamins C, E, Carotenoids and Epicatechin Concentrations}

Vitamins C, E, carotenoid concentrations and epicatechin concentrations in urine as uncorrected, creatinine corrected and volume corrected are presented in Table 4. No significant differences in change between groups were found for vitamins $\mathrm{C}, \mathrm{E}$ or the five carotenoids measured. Epicatechin was not detected in serum samples. For epicatechin detected in urine, there were no significant differences between the groups at week-0. Change in volume-corrected epicatechin was statistically significant between groups $(\mathrm{p}=0.03)$, and this approached significance for uncorrected epicatechin and creatinine-corrected epicatechin values $(\mathrm{p}=0.08$ and $\mathrm{p}=0.06$, respectively). Post-hoc SNK tests indicated that the change in volume-adjusted epicatechin for the apple extract group was significantly larger than for the placebo and the low polyphenol apple groups $(p=0.002$ and $p=0.01$ respectively).

Post-hoc, unplanned analyses explored the main endpoints (oxidised LDL and inflammatory markers) further and ignored original group allocation, but examined these data according to whether a participant had been allocated to a high (high polyphenol apple, epicatechin capsule or freeze dried apple product) or low (low polyphenol apple or control/placebo) epicatechin containing food or product. The reduction in oxidised LDL (week 4 - baseline) was significantly higher in those on the high epicatechin foods or products, compared to those on the low epicatechin containing foods or products (mean (SD) change high epicatechin -7.0 (11.8); low epicatechin 2.5 (15.5); $\mathrm{p}=0.010)$. No significant differences were observed for post prandial oxidised LDL, ICAM-1, VCAM-1, E-selectin or CRP. Change in epicatechin excretion was significantly larger in the high epicatechin groups, compared with the low epicatechin groups, whether unadjusted, creatinine-adjusted or volume adjusted data were used ( $p=0.02,0.009$ and 0.005 , respectively). An alternative approach was to examine the association between the change in epicatechin excretion and the change in endpoint - the change in epicatechin tended to be associated with the change in oxidised LDL, depending on what urine standardisation method was used (change in unstandardized epicatechin $\mathrm{r}=-0.231, \mathrm{p}=0.094$; change in creatinine-corrected epicatechin $\mathrm{r}=-0.218, \quad \mathrm{p}=0.114$; change in volume-corrected epicatechin $\mathrm{r}=-0.255, \mathrm{p}=0.063$; Pearson correlation coefficients). No significant associations were demonstrated for E-selectin, ICAM-1, VCAM-1, hsCRP or post-prandial oxidised LDL.

\subsection{Levels of Liking Questionnaire}

Participants were asked to rate their liking of various foods at weeks 0, 2 and 4 according to a 9-point hedonic scale, which ranged from $1=$ dislike extremely to $9=$ like extremely. Average fruit and vegetable scores and apple scores ranged between 6 and 8, meaning participants liked these foods slightly, moderately or very much. There were no obvious changes over the duration of the intervention in levels of liking of overall fruit and vegetables or apples (data not shown), although formal statistical analysis was not conducted.

\subsection{Tolerability Questionnaire Results}

A total of 5 participants reported side effects: 1 participant taking the high polyphenol apples, the low polyphenol apples and the apple pieces reported side effects, whilst 2 participants taking the placebo capsule reported side effects. The participant taking the low polyphenol apple reported having stomach cramps, whilst the participant taking the apple pieces reported diarrhoea in addition to stomach cramps. The two participants taking the placebo capsule reported different side effects: one noted that their hay fever worsened however, the symptoms subsided approximately an hour after consumption, whilst the other participant complained of needing to use the toilet more frequently. 
Table 3. Change in lipid biomarkers after 4 week intervention with apples and apple products, according to intervention group

\begin{tabular}{|c|c|c|c|c|c|}
\hline & Intervention Group & $N=$ & Week 0 & Week 4 & Change at Week 4 \\
\hline \multirow{6}{*}{$\begin{array}{l}\text { HDL } \\
(\mathrm{mmol} / \mathrm{L})\end{array}$} & Placebo Capsule & 12 & $1.31(0.09)$ & $1.31(0.08)$ & $-0.002(-0.06,0.05)$ \\
\hline & Apple Extract & 12 & $1.43(0.11)$ & $1.44(0.11)$ & $0.02(-0.05,0.09)$ \\
\hline & High Polyphenol Apple & 7 & $1.32(0.08)$ & $1.37(0.11)$ & $0.05(-0.08,0.18)$ \\
\hline & Low Polyphenol Apple & 12 & $1.26(0.08)$ & $1.30(0.07)$ & $0.04(-0.02,0.11)$ \\
\hline & Apple Pieces & 12 & $1.34(0.11)$ & $1.34(0.11)$ & $-0.001(-0.05,0.05)$ \\
\hline & Between Groups P value & & 0.79 & 0.83 & 0.68 \\
\hline \multirow{6}{*}{$\begin{array}{l}\text { Triglycerides } \\
(\mathrm{mmol} / \mathrm{L})\end{array}$} & Placebo Capsule & 12 & $1.07(0.17)$ & $1.13(0.16)$ & $0.06(-0.11,0.23)$ \\
\hline & Apple Extract & 12 & $1.23(0.23)$ & $1.18(0.19)$ & $-0.05(-0.20,0.10)$ \\
\hline & High Polyphenol Apple & 7 & $1.04(0.16)$ & $1.35(0.34)$ & $0.31(-0.24,0.86)$ \\
\hline & Low Polyphenol Apple & 12 & $1.26(0.20)$ & $1.14(2.00)$ & $-0.11(-0.29,0.06)$ \\
\hline & Apple Pieces & 12 & $1.33(0.16)$ & $1.36(0.16)$ & $0.03(-0.25,0.30)$ \\
\hline & Between Groups P value & & 0.80 & 0.86 & 0.16 \\
\hline \multirow{6}{*}{$\begin{array}{l}\text { Cholesterol } \\
(\mathrm{mmol} / \mathrm{L})\end{array}$} & Placebo Capsule & 12 & $4.75(0.26)$ & $4.808(0.256)$ & $0.06(-0.25,0.37)$ \\
\hline & Apple Extract & 12 & $4.84(0.28)$ & $4.754(0.284)$ & $-0.09(-0.41,0.23)$ \\
\hline & High Polyphenol Apple & 7 & $4.49(0.32)$ & $4.736(0.288)$ & $0.25(-0.24,0.74)$ \\
\hline & Low Polyphenol Apple & 12 & $4.32(0.23)$ & $4.491(0.270)$ & $0.17(-0.16,0.49)$ \\
\hline & Apple Pieces & 12 & $5.51(0.41)$ & $5.578(0.338)$ & $0.07(-0.23,0.38)$ \\
\hline & Between Groups P value & & 0.07 & 0.09 & 0.64 \\
\hline \multirow{6}{*}{$\begin{array}{l}\text { Oxidised } L D L \\
(U / L)\end{array}$} & Placebo Capsule & 12 & $35.0(1.73)$ & $34.97(4.962)$ & $0.01(-9.92,9.94)$ \\
\hline & Apple Extract & 12 & $40.2(4.11)$ & $30.60(2.361)$ & $-9.62(-16.2,-3.00)$ \\
\hline & High Polyphenol Apple & 7 & $35.8(4.64)$ & $30.54(2.487)$ & $-5.28(-15.1,4.5)$ \\
\hline & Low Polyphenol Apple & 12 & $31.0(2.64)$ & $35.89(5.885)$ & $4.93(-5.01,14.9)$ \\
\hline & Apple Pieces & 12 & $45.6(5.25)$ & $39.15(4.357)$ & $-5.22(-14.8,4.31)$ \\
\hline & Between Groups P value & & 0.07 & 0.64 & 0.12 \\
\hline \multirow{6}{*}{$\begin{array}{l}\text { Oxidised LDL } \\
\text { (Post-Prandial) } \\
\text { (U/L) }\end{array}$} & Placebo Capsule & 12 & $34.6(4.48)$ & $29.6(3.48)$ & $-5.03(-16.3,6.25)$ \\
\hline & Apple Extract & 12 & $30.1(2.02)$ & $28.9(3.00)$ & $-1.39(-6.68,3.91)$ \\
\hline & High Polyphenol Apple & 7 & $38.2(12.9)$ & $24.8(3.11)$ & $-16.9(-74.2,40.5)$ \\
\hline & Low Polyphenol Apple & 12 & $22.6(2.75)$ & $25.1(2.69)$ & $2.25(-1.57,6.10)$ \\
\hline & Apple Pieces & 12 & $32.7(4.81)$ & $40.6(10.6)$ & $6.13(-22.0,34.3)$ \\
\hline & Between Groups P value & & 0.32 & 0.26 & 0.40 \\
\hline \multirow{6}{*}{$\begin{array}{l}\text { hsCRP } \\
(\mu g / d L)\end{array}$} & Placebo capsule & 12 & $114(46.1)$ & $129(41.7)$ & $15.5(-30.9,61.3)$ \\
\hline & Apple Extract & 12 & $103(24.0)$ & $135(42.2)$ & $31.8(-34.9,98.4)$ \\
\hline & High Polyphenol Apple & 7 & $224(124)$ & $242(112)$ & $-21.5(-73.0,30.0)$ \\
\hline & Low Polyphenol Apple & 12 & $246(65.0)$ & $168(65.4)$ & $-77.2(-185,30.3)$ \\
\hline & Apple Pieces & 12 & $197(34.3)$ & $237(56.1)$ & $40.1(-28.2,109)$ \\
\hline & Between Groups P value & & 0.26 & 0.66 & 0.09 \\
\hline \multirow{6}{*}{$\begin{array}{l}\text { E Selectin } \\
(\mathrm{ng} / \mathrm{mL})\end{array}$} & Placebo capsule & 12 & $44.4(4.05)$ & $44.9(4.58)$ & $0.55(-2.27,3.37)$ \\
\hline & Apple Extract & 12 & $41.8(4.05)$ & $42.1(3.02)$ & $0.33(-2.73,3.38)$ \\
\hline & High Polyphenol Apple & 7 & $36.3(3.87)$ & $36.4(3.30)$ & $0.07(-3.14,3.28)$ \\
\hline & Low Polyphenol Apple & 12 & $43.4(5.79)$ & $45.4(5.22)$ & $2.01(-1.64,5.66)$ \\
\hline & Apple Pieces & 12 & $44.4(3.17)$ & $48.4(3.43)$ & $3.15(0.18,6.11)$ \\
\hline & Between Groups P value & & 0.82 & 0.45 & 0.53 \\
\hline \multirow{6}{*}{$\begin{array}{l}I C A M-1 \\
(n g / m L)\end{array}$} & Placebo capsule & 12 & $241(17.2)$ & $245(17.3)$ & $4.17(-22.6,30.9)$ \\
\hline & Apple Extract & 12 & $213(16.6)$ & $223(21.6)$ & $9.97(-21.8,41.8)$ \\
\hline & High Polyphenol Apple & 7 & $230(19.9)$ & $232(24.7)$ & $2.14(-17.2,21.5)$ \\
\hline & Low Polyphenol Apple & 12 & $245(21.1)$ & $242(25.4)$ & $-2.33(-20.7,16.1)$ \\
\hline & Apple Pieces & 12 & $260(14.5)$ & $231(26.1)$ & $-26.1(-95.4,43.2)$ \\
\hline & Between Groups P value & & 0.43 & 0.96 & 0.64 \\
\hline \multirow{6}{*}{$\begin{array}{l}V C A M-1 \\
(n g / m L)\end{array}$} & Placebo capsule & 12 & $662(24.9)$ & $709(47.0)$ & $47.3(-33.6,128)$ \\
\hline & Apple Extract & 12 & $626(21.8)$ & $616(18.3)$ & $-9.75(-28.3,8.81)$ \\
\hline & High Polyphenol Apple & 7 & $671(31.7)$ & $655(35.1)$ & $-15.3(-62.4,31.9)$ \\
\hline & Low Polyphenol Apple & 12 & $622(33.2)$ & $601(36.9)$ & $-21.0(-62.5,20.5)$ \\
\hline & Apple Pieces & 12 & $679(38.3)$ & $707(30.9)$ & $30.5(2.88,58.0)$ \\
\hline & Between Groups P value & & 0.56 & 0.06 & 0.12 \\
\hline
\end{tabular}

Absolute variables are presented as mean (SD), change at week 4 presented as mean $(95 \% \mathrm{CI})$. Baseline values and changes were compared between groups using one way ANOVA with SNK post-hoc tests. Presentation of mean change $(95 \% \mathrm{CI})$ allows examination of any statistically significant within group changes (i.e. any confidence intervals that do not cross 1.0 ). 
Table 4. Change in serum and urine antioxidant status after 4 week intervention with apples and apple products, according to intervention group

\begin{tabular}{|c|c|c|c|c|c|}
\hline & Intervention Group & $N=$ & Week 0 & Week 4 & Change at Week 4 \\
\hline \multirow{6}{*}{$\begin{array}{l}\text { Lutein } \\
(\mu \mathrm{mol} / \mathrm{L})\end{array}$} & Placebo Capsule & 12 & $0.192(0.07)$ & $0.207(0.07)$ & $0.016(0.001,0.031)$ \\
\hline & Apple Extract & 12 & $0.180(0.05)$ & $0.190(0.04)$ & $0.010(-0.006,0.026)$ \\
\hline & High Polyphenol Apple & 7 & $0.215(0.08)$ & $0.227(0.12)$ & $0.012(-0.017,0.041)$ \\
\hline & Low Polyphenol Apple & 12 & $0.151(0.08)$ & $0.139(0.06)$ & $-0.012(-0.053,0.029)$ \\
\hline & Apple Pieces & 12 & $0.205(0.10)$ & $0.189(0.07)$ & $-0.016(-0.067,0.035)$ \\
\hline & Between Groups P value & & 0.39 & 0.07 & 0.47 \\
\hline \multirow{5}{*}{$\begin{array}{l}\text { Zeaxanthin } \\
(\mu \mathrm{mol} / \mathrm{L})\end{array}$} & Apple Extract & 12 & $0.048(0.02)$ & $0.055(0.03)$ & $0.007(0.00,0.013)$ \\
\hline & High Polyphenol Apple & 7 & $0.049(0.02)$ & $0.053(0.03)$ & $0.004(-0.006,0.013)$ \\
\hline & Low Polyphenol Apple & 12 & $0.039(0.01)$ & $0.039(0.02)$ & $0.000(-0.005,0.005)$ \\
\hline & Apple Pieces & 12 & $0.049(0.02)$ & $0.048(0.02)$ & $0.000(-0.012,0.011)$ \\
\hline & Between Groups P value & & 0.54 & 0.39 & 0.47 \\
\hline \multirow{5}{*}{$\begin{array}{l}\text { B-Cryptoxanthin } \\
(\mu \mathrm{mol} / \mathrm{L})\end{array}$} & Placebo Capsule & 12 & $0.116(0.09)$ & $0.129(0.09)$ & $0.013(-0.017,0.042)$ \\
\hline & High Polyphenol Apple & 7 & $0.064(0.03)$ & $0.062(0.03)$ & $-0.003(-0.019,0.014)$ \\
\hline & Low Polyphenol Apple & 12 & $0.072(0.06)$ & $0.050(0.02)$ & $-0.023(-0.059,0.013)$ \\
\hline & Apple Pieces & 12 & $0.093(0.07)$ & $0.090(0.07)$ & $-0.003(-0.016,0.01)$ \\
\hline & Between Groups P value & & 0.24 & 0.04 & 0.67 \\
\hline \multirow{6}{*}{$\begin{array}{l}\alpha \text {-Carotene } \\
(\mu \mathrm{mol} / \mathrm{L})\end{array}$} & Placebo Capsule & 12 & $0.245(0.20)$ & $0.275(0.23)$ & $29.9(-44.0,64.2)$ \\
\hline & Apple Extract & 12 & $0.149(0.06)$ & $0.170(0.06)$ & $21.3(-9.02,51.7)$ \\
\hline & High Polyphenol Apple & 7 & $0.126(0.05)$ & $0.119(0.04)$ & $-7.13(-47.3,33.1)$ \\
\hline & Low Polyphenol Apple & 12 & $0.112(0.06)$ & $0.124(0.05)$ & $11.2(-28.0,50.4)$ \\
\hline & Apple Pieces & 12 & $0.151(0.09)$ & $0.153(0.07)$ & $2.00(-43.4,47.4)$ \\
\hline & Between Groups P value & & 0.06 & 0.02 & 0.63 \\
\hline \multirow{4}{*}{$\begin{array}{l}\text { B-Carotene } \\
(\mu \mathrm{mol} / \mathrm{L})\end{array}$} & Placebo Capsule & 12 & $0.638(0.54)$ & $0.668(0.64)$ & $0.030(-0.111,0.017)$ \\
\hline & Apple Extract & 12 & $0.478(0.27)$ & $0.509(0.26)$ & $0.031(-0.072,0.133)$ \\
\hline & High Polyphenol Apple & 7 & $0.504(0.33)$ & $0.415(0.21)$ & $-0.089(-0.240,0.061)$ \\
\hline & Low Polyphenol Apple & 12 & $0.316(0.18)$ & $0.340(0.17)$ & $0.024(-0.036,0.083)$ \\
\hline \multirow{6}{*}{ Epicatechin $(\mu \mathrm{mol} / \mathrm{L})$} & Placebo Capsule & 12 & $592(215)$ & $418(119)$ & $-175(-659,310)$ \\
\hline & Apple Extract & 12 & $614(128)$ & $3482(1058)$ & $2868(489,5247)$ \\
\hline & High Polyphenol Apple & 7 & $425(103)$ & $2191(648)$ & $1766(195,3337)$ \\
\hline & Low Polyphenol Apple & 12 & $689(188)$ & $1253(339)$ & $563(-456,1582)$ \\
\hline & Apple Pieces & 12 & $1116(413)$ & $2422(912)$ & $1305(-1043,3654)$ \\
\hline & Between Groups P value & & 0.42 & 0.04 & 0.08 \\
\hline \multirow{6}{*}{$\begin{array}{l}\text { Creatinine Corrected } \\
\text { Epicatechin } \\
\text { (nmoles/mgcreat) }\end{array}$} & Placebo Capsule & 12 & $15.6(6.32)$ & $10.8(5.48)$ & $-4.81(-21.7,12.1)$ \\
\hline & Apple Extract & 12 & $10.9(2.81)$ & $77.8(27.4)$ & $66.6(9.43,124)$ \\
\hline & High Polyphenol Apple & 7 & $6.44(2.21)$ & $31.5(12.9)$ & $25.0(-3.83,53.9)$ \\
\hline & Low Polyphenol Apple & 12 & $14.6(4.30)$ & $13.8(3.53)$ & $1.23(-10.9,13.4)$ \\
\hline & Apple Pieces & 12 & $13.9(5.00)$ & $50.2(27.0)$ & $36.3(-23.8,96.4)$ \\
\hline & Between Groups P value & & 0.78 & 0.08 & 0.06 \\
\hline \multirow{6}{*}{$\begin{array}{l}\text { Volume Corrected } \\
\text { Epicatechin } \\
\text { (nmoles/sample) }\end{array}$} & Placebo Capsule & 12 & $497(254)$ & $255(116)$ & $-242(-799,315)$ \\
\hline & Apple Extract & 12 & $371(115)$ & $2025(595)$ & $1655(299,3010)$ \\
\hline & High Polyphenol Apple & 7 & $190(65.3)$ & $804(185)$ & $614(195,1032)$ \\
\hline & Low Polyphenol Apple & 12 & $253(73.1)$ & $358(123)$ & $106(-202,413)$ \\
\hline & Apple Pieces & 12 & $373(160)$ & $1156(527)$ & $783(-459,2025)$ \\
\hline & Between Groups P value & & 0.75 & 0.01 & 0.03 \\
\hline
\end{tabular}

Absolute variables are presented as mean (SD), change at week 4 presented as mean $(95 \% \mathrm{CI})$.

In relation to ease of consumption, the majority of the participants rated the study products very easy, easy or sometimes hard and sometimes easy to consume. However, $57 \%$ of participants found it hard to consume the high polyphenol apples. Participants commented that they were dry, strong in flavour and extra foods were required to eat along with the apples to ease consumption. One participant also reported a feeling of fullness and found that, on occasions, heartburn developed after eating the apples in the evening. One participant in the apple pieces group found consumption hard, as it was difficult to remember to take the apple pieces routinely and also difficult to incorporate into the diet.

The majority of participants ( $\mathrm{n}=51$ across all groups) rated acceptability of study products as very acceptable or acceptable if it were to improve their heart health. However, three participants taking the high polyphenol apples and one participant taking the low polyphenol apples rated acceptability as unsure.

Responses for willingness to continue post intervention varied, however, only one participant taking the high polyphenol apples stated they strongly agreed. Between 4 
and 5 participants strongly agreed across all other study groups. At least one participant in each group was unsure if they would be willing to consume the products post-intervention. From participants who were unwilling to continue consumption of the study products, reasons included: prefer to improve their overall diet and lifestyle as opposed to taking capsules; those on apple pieces or 2 apples per day said that they were "fed up" consuming the apples by the end of the intervention period (low polyphenol apples), and they didn't like the taste/texture (apple pieces). Results varied similarly for willingness to purchase study products. The majority of participants would be willing to consume/purchase the products post intervention and either strongly agreed or agreed with the statement. At least one participant in each of the intervention groups was unsure if or disagreed that they would be willing to purchase the products post-intervention; with two participants in the high polyphenol apple group (56\%) saying they were unsure or disagreed. Additionally, one participant receiving the encapsulated apple extract strongly disagreed that they would purchase the product post-intervention, as did one participant receiving the apple pieces.

\section{Discussion}

The APPS study examined the feasibility of conducting a study to test the effect of polyphenol-rich apples, apple extract and freeze dried apple pieces (with similar epicatechin content) on risk factors and biomarkers for CVD, in a pilot intervention study of participants at elevated risk of CVD.

There was no significant effect of the intervention on weight, BMI, waist and hip circumference or systolic and diastolic blood pressure, although this pilot study phase was unlikely to be appropriately powered. No other studies have directly examined the effect of apples or isolated epicatechin on blood pressure or other CVD risk factors. Weichselbaum et al. [15] reviewed three studies, which had examined the effect of polyphenols, including those found in apples, on blood pressure. Two studies examined quercetin capsules (150mg/day [24] and 730mg/day [25]), and one study examined a milk chocolate drink $(150 \mathrm{mg} /$ day flavanols [26]), containing epicatechin, and each found beneficial effects on blood pressure measurements. The combination of polyphenols in these studies is not directly comparable with the current study. However, it may also be that the dose of epicatechin given in the current study (80mg/day) was not high enough to cause a beneficial effect, and this would need further examination in appropriately powered and designed studies.

The effect of apple consumption and epicatechin/polyphenol levels on oxLDL have previously been investigated by a small number of studies. In a study by Serra et al [27], rats were given one of three varieties of apple, a cholesterol enriched diet or a controlled diet. All three types of apple were found to significantly reduce levels of oxLDL when compared to either of the diets. The study also concluded that a number of phytochemicals, included epicatechin, were responsible for the cholesterol lowering effect observed. Following on from this, Zhao et al [28] conducted a randomised controlled trial in 41 participants, who received either whole apple, a polyphenol capsule or a placebo capsule. The study reported that apples and the polyphenol capsule significantly reduced concentrations of oxidised low-density lipoprotein/beta 2 -glycoprotein complex $\left(\mathrm{OxLDL}-\beta_{2} \mathrm{GPI}\right)$, which is involved in arthrosclerosis.

In relation to inflammatory markers, evidence is conflicting. Codner-Franch et al [29] found that hs-CRP was significantly reduced in 41 obese children who consumed dried apple pieces enriched with mandarin juice. In a crossover study by Ravn-Haren et al [30] the effect of whole apple, apple pomace, clear and cloudy juices was investigated in 23 healthy participants. No significant differences in CRP were reported. Other inflammatory markers have also been investigated in relation to consumption of cocoa, which is high in polyphenols, with similarly mixed results [31]. The current study did not find a significant difference in inflammatory markers following the intervention. It is unclear as to why results are conflicting, but, again, this pilot study was not powered for these outcomes.

In relation to dietary intakes, there were no significant differences at baseline between the intervention groups, and the only nutrient where the change was statistically significant between groups was dietary fibre. In a 6-week intervention trial by Hyson et al. [32], where participants $(n=25)$ consumed $340 \mathrm{~g}$ of whole apple daily, similarly to the current study, the only nutrient to change was dietary fibre, which was increased The lack of significant change in nutrient intakes (apart from dietary fibre), may be expected, as eating two apples or following a low apple diet whilst consuming a capsule or freeze-dried apple pieces is unlikely to have made a major difference to other parts of the participants' normal diet.

No epicatechin was detected in serum samples. This may be explained by the dose of epicatechin given in the current study (80mg/day) not being high enough to cause an increase, however, this would need further examination, as the same apple extract increased plasma epicatechin concentrations in another study when provided at $70 \mathrm{mg}$ and $140 \mathrm{mg}$ as a single dose [33]. Epicatechin was detected in participants' urine, and showed between group differences in change in excretion that were significant or approached significance, when corrected for urinary volume or creatinine content. It has been recommended that urinary polyphenol excretion should be expressed as volume corrected, as well as creatinine corrected, as volume-corrected results may be a better biomarker for polyphenol dietary intake, in the absence of covariate adjustment [34]. All groups receiving an apple product in the study showed increased epicatechin concentrations in urine after 4 weeks, with those receiving the apple extract seeing the greatest increase, indicating good compliance in terms of consumption of the apple products.

However, with respect to tolerability, some of the participants in both of the apple groups (i.e. low and high polyphenol) commented post intervention that they were happy not to have to eat any more apples. Furthermore, $57 \%$ of the high polyphenol apple group found it hard to consume the 2 apples per day for the 4 week intervention period. Generally in the other intervention groups, consumption of the products was found to be very easy or easy. It may be that the participants did not consider the high polyphenol apples, as the intervention study product, to be representative of the "apples" food group in general, 
when completing the level of liking questionnaire, which rated the level of liking of apples as being relatively high.

The majority of participants in each group (both intervention groups and control), with the exception of those receiving the high polyphenol apples, strongly agreed or agreed that they would be willing to continue consumption of apples/apple products if it improved their heart health. A small number disagreed or strongly disagreed in each group, with the remaining participants stating they were unsure. Some participants indicated that they would prefer to improve their diet/lifestyle as opposed to taking apples or apple products. Although participants have stated that they would prefer to improve their general lifestyle, it is recognised that populations as a whole are not meeting lifestyle targets (e.g. fruit and vegetable targets), according to recent NDNS data [6], and therefore, there still remains a major public health challenge in targeting messages in effective ways to change behaviour.

\subsection{Strengths and Limitations of the APPS Study}

There were a number of strengths to the APPS study. Firstly, general strengths were: it was a randomised controlled intervention; apples were supplied to participants to ensure limited costs were incurred by the participants; advice and support was given at the beginning and throughout the study to ensure participants understood fully the requirements for involvement. Secondly, multiple methods were used to encourage good participant compliance and to monitor compliance and minimise dropouts over the course of the study: for example, participants were contacted by telephone on a weekly basis; 4-day diet diaries were collected at the start and end of the study; participants received a weekly delivery of apples to a place of convenience; a telephone number for the study researcher was provided to participants; $£ 30$ was given to participants on successful completion of the study to cover travel expenses to the study visits, ensuring no participant was out of pocket during the course of the study. Thirdly, participants had limited restrictions placed on them throughout the study (apart from those who had to consume a low apple diet); therefore, requirements for the study were not intensive so it would have been relatively easy for participants to stick to the diet. Finally, the epicatechin content of study products was standardised for all intervention groups and the study was placebo-controlled and double blinded in terms of the apple extract versus placebo comparison, while for the postprandial element of the study, participants were given a standardised breakfast.

However, the APPS study also had limitations: firstly, establishing accurate dietary intake data from intervention studies is difficult, as participants tend to under-report foods which are high in fat and over-report foods, such as fruit and vegetables. Although 4-day diet diaries were collected at the start and end of the study to ascertain participants' dietary intakes, misreporting was likely to have occurred. Furthermore, participants may have changed their intake of foods whilst completing the diet diaries, for example, to ease recording of foods consumed or to make their diet seem healthier.

For future studies, there are a number of considerations. The availability of the high polyphenol apples was limited, and their storage without deterioration challenging, which may be a problem in a larger scale definitive study. Additionally, a high proportion of participants were unsure if they would continue eating the high polyphenol apples in comparison to the other intervention groups. This was in part due to the taste and texture. Apple extract capsules fared best in terms of acceptability and willingness to continue/purchase items post-intervention, although some participants also felt that improving overall diet and lifestyle would be more beneficial than taking capsules or apple products.

\section{Conclusions}

In conclusion, the APPS study explored the feasibility of conducting an intervention study using different apples and apple products on CVD risk factors. In terms of exploring likely risk factors modifiable by apple consumption, this pilot study found no statistically significant effect on oxidised LDL levels after consumption of epicatechin-rich apples, apple extract, or freeze-dried apple pieces, in participants at elevated risk of CVD. However, the change in epicatechin excretion tended to be associated with change in oxidised LDL, while a comparison of the high versus low epicatechin containing foods also appeared to show a possible benefit on fasting oxidised LDL, therefore a larger study powered to demonstrate such an effect would be of interest. Due to the low sample size and pilot design of the study, a retrospective power calculation based on this study could inform the design of a larger intervention study, which would be required to examine if apples and apple products have an effect on oxidised LDL cholesterol and indeed CVD risk, as evidence from current studies is extremely limited. In addition, the study has provided information on tolerability and willingness to consume/purchase the various study products. Given the low fruit and vegetable intake of the UK population, products which remove barriers to consumption could well have an impact on population consumption levels, but their health benefits still require further exploration.

\section{Acknowledgements}

The researchers would like to thank Coressence, Bristol, UK for their provision of apple products used in the study. The researchers would also like to thank Cathy Pogue for her assistance with lab analysis.

\section{Statement of Competing Interests}

$\mathrm{OH}$ and KT are employed by DuPont. The authors declare no other conflict of interest regarding this study.

\section{List of Abbreviations}

$\mathrm{ACN}$ - acetonitrile

ANOVA - one-way analysis of variance

APPS - Apple/Apple Pieces Study

BMI - body mass index 
CRP - C-reactive protein

$\mathrm{CV}$ - coefficient of variance

CVD - cardiovascular disease

ELISA - enzyme-linked immunoassay

EPIC - European prospective investigation of cancer

FFQ - food frequency questionnaire

HDL - high density lipoprotein

HPLC-MS/MS - high performance liquid

chromatograph tandem mass spectrometry

ICAM-1 - intercellular adhesion molecule-1

LDL - low density lipoprotein

LOQ - limit of quantification

$\mathrm{QC}$ - quality control

$\mathrm{RCT}$ - randomised controlled trial

RPM - revolutions per minute

SNK - Student Newman Keuls

SPSS - Statistical program for social sciences

UK - United Kingdom

USA - United States of America

VCAM-1 - vascular cell adhesion molecule-1

\section{References}

[1] Wang X, Ouyang Y, Liu J, Zhu M, Zhao G, Bao W, et al. Fruit and vegetable consumption and mortality from all causes, cardiovascular disease, and cancer: systematic review and doseresponse meta-analysis of prospective cohort studies. BMJ 2014 Jul 29; 349: g4490.

[2] Zhan J, Liu Y, Cai L, Xu F, Xie T, He Q. Fruit and vegetable consumption and risk of cardiovascular disease: a meta-analysis of prospective cohort studies. Crit Rev Food Sci Nutr 2017; 57(8): 1650-1663.

[3] McCall DO, McGartland CP, McKinley MC, Patterson CC, Sharpe P, McCance DR, et al. Dietary intake of fruits and vegetables improves microvascular function in hypertensive subjects in a dose-dependent manner. Circulation 2009 Apr 28; 119(16): 2153-2160.

[4] Appel LJ, Moore TJ, Obarzanek E, Vollmer WM, Svetkey LP, Sacks FM, et al. A clinical trial of the effects of dietary patterns on blood pressure. N Engl J Med 1997; 336(16): 1117-1124.

[5] Hall JN, Moore S, Harper SB, Lynch JW. Global variability in fruit and vegetable consumption. Am J Prev Med 2009 May; 36(5): 402-409.e5.

[6] Public Health England. National Diet and Nutrition Survey: Results from Years 5 and 6 (combined) of th Rolling Progamme (2012/2013 - 2013/2014). 2016.

[7] Appleton KM, McGill R, Neville C, Woodside JV. Barriers to increasing fruit and vegetable intakes in the older population of Northern Ireland: low levels of liking and low awareness of current recommendations. Public Health Nutr 2010; 13(4): 514-521.

[8] Glasson C, Chapman K, James E. Fruit and vegetables should be targeted separately in health promotion programmes: differences in consumption levels, barriers, knowledge and stages of readiness for change. Public Health Nutr 2011 Apr; 14(4): 694-701.

[9] Schatzer M, Rust P, Elmadfa I. Fruit and vegetable intake in Austrian adults: intake frequency, serving sizes, reasons for and barriers to consumption, and potential for increasing consumption. Public Health Nutr 2010 Apr; 13(4): 480-487.

[10] Haynes-Maslow L, Parsons SE, Wheeler SB, Leone LA. A qualitative study of perceived barriers to fruit and vegetable consumption among low-income populations, North Carolina, 2011. Prev Chronic Dis 2013; 10: E34.

[11] Williamson G, Holst B. Dietary reference intake (DRI) value for dietary polyphenols: are we heading in the right direction? $\mathrm{Br} \mathrm{J}$ Nutr 2008; 99(S3): S55-S58.

[12] Chong MF, Macdonald R, Lovegrove JA. Fruit polyphenols and CVD risk: a review of human intervention studies. Br J Nutr 2010; 104(S3): S28-S39.
[13] Ceymann M, Arrigoni E, Schärer H, Nising $A B$, Hurrell RF. Identification of apples rich in health-promoting flavan-3-ols and phenolic acids by measuring the polyphenol profile. Journal of Food Composition and Analysis 2012; 26(1): 128-135.

[14] Jensen EN, Buch-Andersen T, Ravn-Haren G, Dragsted LO. Minireview: The effects of apples on plasma cholesterol levels and cardiovascular risk-a review of the evidence. The Journal of Horticultural Science and Biotechnology 2009; 84(6): 34-41.

[15] Weichselbaum E, Wyness L, Stanner S. Apple polyphenols and cardiovascular disease-a review of the evidence. Nutr Bull 2010; 35(2): 92-101.

[16] Boyer J, Liu RH. Apple phytochemicals and their health benefits. Nutrition journal 2004; 3(1): 5.

[17] Craft NE. Carotenoid reversed-phase high-performance liquid chromatography methods: Reference compendium. Meth Enzymol 1992; 213: 185-205.

[18] Vuilleumier J, Keck E. Fluorometric assay of vitamin C in biological materials using a centrifugal analyser with fluorescence attachment. Journal of Micronutrient Analysis 1989.

[19] Nielsen SE, Freese R, Kleemola P, Mutanen M. Flavonoids in human urine as biomarkers for intake of fruits and vegetables. Cancer Epidemiol Biomarkers Prev 2002 May; 11(5): 459-466.

[20] Rein D, Lotito S, Holt RR, Keen CL, Schmitz HH, Fraga CG. Epicatechin in human plasma: in vivo determination and effect of chocolate consumption on plasma oxidation status. J Nutr 2000 Aug; 130(8S Suppl): 2109S-14S.

[21] Richelle M, Tavazzi I, Enslen M, Offord EA. Plasma kinetics in man of epicatechin from black chocolate. Eur J Clin Nutr 1999 Jan; 53(1): 22-26.

[22] EPIC-Norfolk Food Frequency Questionnaire. 2017; Available at: http: //www.srl.cam.ac.uk/epic/epicffq/. Accessed August, 2017.

[23] Ried K, Frank OR, Stocks NP. Dark chocolate or tomato extract for prehypertension: a randomised controlled trial. BMC complementary and alternative medicine 2009; 9(1): 22 .

[24] Egert S, Bosy-Westphal A, Seiberl J, Kürbitz C, Settler U, Plachta-Danielzik S, et al. Quercetin reduces systolic blood pressure and plasma oxidised low-density lipoprotein concentrations in overweight subjects with a high-cardiovascular disease risk phenotype: a double-blinded, placebo-controlled cross-over study. Br J Nutr 2009; 102(7): 1065-1074.

[25] Edwards RL, Lyon T, Litwin SE, Rabovsky A, Symons JD, Jalili T. Quercetin reduces blood pressure in hypertensive subjects. J Nutr 2007 Nov; 137(11): 2405-2411.

[26] Fraga CG, Actis-Goretta L, Ottaviani JI, Carrasquedo F, Lotito SB, Lazarus S, et al. Regular consumption of a flavanol-rich chocolate can improve oxidant stress in young soccer players. Journal of Immunology Research 2005; 12(1): 11-17.

[27] Serra AT, Rocha J, Sepodes B, Matias AA, Feliciano RP, de Carvalho A, et al. Evaluation of cardiovascular protective effect of different apple varieties - Correlation of response with composition. Food Chemistry 201215 December 2012; 135(4): 2378-2386.

[28] Zhao S, Bomser J, Joseph EL, DiSilvestro RA. Intakes of apples or apple polyphenols decease plasma values for oxidized lowdensity lipoprotein/beta 2-glycoprotein I complex. Journal of Functional Foods 2013; 5(1): 493-497.

[29] Codoner-Franch P, Betoret E, Betoret N, Lopez-Jaen AB, VallsBelles V, Fito P. Dried apples enriched with mandarin juice by vacuum impregnation improve antioxidant capacity and decrease inflammation in obese children. Nutr Hosp 2013 Jul-Aug; 28(4): 1177-1183.

[30] Ravn-Haren G, Dragsted LO, Buch-Andersen T, Jensen EN, Jensen RI, Nemeth-Balogh M, et al. Intake of whole apples or clear apple juice has contrasting effects on plasma lipids in healthy volunteers. Eur J Nutr 2013 Dec; 52(8): 1875-1889.

[31] Khan N, Khymenets O, Urpí-Sardà M, Tulipani S, Garcia-Aloy M, Monagas $\mathrm{M}$, et al. Cocoa polyphenols and inflammatory markers of cardiovascular disease. Nutrients 2014; 6(2): 844-880.

[32] Hyson D, Studebaker-Hallman D, DAVIS PA, GERSHWIN ME. Apple juice consumption reduces plasma low-density lipoprotein oxidation in healthy men and women. Journal of medicinal food 2000; 3(4): 159-166.

[33] Hollands WJ, Hart DJ, Dainty JR, Hasselwander O, Tiihonen K, Wood R, et al. Bioavailability of epicatechin and effects on nitric oxide metabolites of an apple flavanol-rich extract supplemented 
beverage compared to a whole apple puree: a randomized, placebo-controlled, crossover trial. Molecular nutrition \& food research 2013; 57(7): 1209-1217.

[34] Zamora-Ros R, Rabassa M, Cherubini A, Urpi-Sarda M, Llorach
$\mathrm{R}$, Bandinelli S, et al. Comparison of 24-h volume and creatininecorrected total urinary polyphenol as a biomarker of total dietary polyphenols in the Invecchiare InCHIANTI study. Anal Chim Acta 2011; 704(1): 110-115.

\section{Supplementary}

\section{HPLC-MS/MS detection and quantification of epicatcechin in urine and serum}

\section{Sample preparation}

For each serum and urine, samples were run in three batches, in consecutive numerical order (containing baseline and week 4 samples simultaneously for each participant), the analyst was blinded to study group. Serum samples were analysed in duplicate, while urine samples were analysed singly, all samples were spiked with $100 \mathrm{ng} / \mathrm{ml}$ of fisetin for use as internal standard. $100 \mu \mathrm{l}$ of each sample underwent enzymatic hydrolysis by adding $\sim 100$ units of sulphatase and $\sim 4,000$ units of ß-glucuronidase (both from Helix pomatia, type H-1) suspended in $0.1 \mathrm{~mol} / \mathrm{L}$ acetate buffer (pH 5.0) and incubating (45 minutes; $37^{\circ} \mathrm{C}$ ). After incubation, $20 \mu \mathrm{l}$ of fisetin in methanol $(\mathrm{MeOH})$ was spiked into serum samples, which were then de-proteinised with $280 \mu$ l acetonitrile (ACN) containing $3 \mathrm{~g} / \mathrm{L}$ tert-butylhydroquinone (TBHQ). Urine samples after incubation, underwent a 3 hour freeze at $-80^{\circ} \mathrm{C}$, and were freeze dried overnight for $\sim 19$ hours on a Thermo Savant ModulyoD-230 (Thermo Scientific, West Sussex, UK) and reconstituted with $400 \mu \mathrm{MeOH}$ containing fisetin. All samples were vortexed $(1 \mathrm{~min})$ and centrifuged $(16,000 x \mathrm{~g} ; 20 \mathrm{~min})$. Serum samples underwent an additional $1: 2$ dilution with ACN (3 g/L TBHQ) to minimise matrix effects and were vortexed (1 min) and centrifuged (16,000 $x \mathrm{~g} ; 20$ min) for a second time. Resulting supernatants were injected directly onto the HPLC.

\section{Quantification by HPLC-MS/MS}

$5 \mu \mathrm{l}$ injections were performed on an Agilent Technologies 1100 series HPLC (Agilent Technologies, Stockport, UK) directly linked to a Waters Micromass Quattro Ultima Platinum API triple quadrupole mass spectrometer (Waters, Dublin, Ireland). The HPLC was a binary system, with a 100 well auto sampler held at room temperature $\left(21^{\circ} \mathrm{C}\right)$.

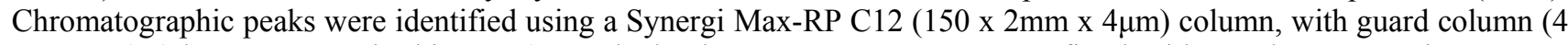
$\mathrm{x} 2.0 \mathrm{~mm}$ ) (Phenomenex, Cheshire, UK) attached. The mass spectrometer was fitted with an electrospray ion source, which was operated in negative ion mode. Optimal MS operating parameters were as follows; capillary voltage $2.75 \mathrm{kV}$; source temperature $120^{\circ} \mathrm{C}$; cone gas flow rate $64 \mathrm{~L} / \mathrm{h}$; desolvation gas flow rate $506 \mathrm{~L} / \mathrm{h}$; desolvation temperature $350^{\circ} \mathrm{C}$. Mobile phase consisted of $95 \% \mathrm{ACN}, 5 \%$ water and $0.1 \%$ formic acid in channel A and $95 \%$ water, $5 \%$ ACN and $0.1 \%$ formic acid in channel $\mathrm{B}$, at a flow rate of $0.2 \mathrm{ml} / \mathrm{min}$. Run time for each sample was $38.2 \mathrm{~min}$. The HPLC system was scheduled to hold $95 \%$ channel B for $1 \mathrm{~min}$, ramping to $5 \%$ channel B over a period of $8 \mathrm{~min}$. This $5 \%$ channel B was held for $7 \mathrm{~min}$, followed by an increase in gradient back to $95 \%$ channel B over $2 \mathrm{~min}$, which was held for 20.2 min to equilibrate the system, prior to the next injection. Limit of quantification (LOQ) for the assay was $11.1 \mathrm{ng} / \mathrm{ml}$.

\section{Serum}

For the serum-QC, mean inter-assay CV was $18.0 \%$ whilst the intra-assay CV was $16.3 \%$. A low epicatechin lithium heparin plasma sample was used as the matrix into which epicatechin was spiked in order to produce a calibration curve ranging from $0-400 \mathrm{ng} / \mathrm{ml}$. Three serum samples per batch were randomly selected and spiked with $300 \mathrm{ng} / \mathrm{ml}$ of epicatechin to assess recovery (recovery $=84.2 \%$ ).

\section{Quality control, calibration curves and recovery}

Matrix-matched QC, standards and samples spiked to assess percent recovery underwent appropriate sample preparation (as described above) for serum and urine. For each batch a fresh calibration curve was prepared and run at the beginning and end of each run.

\section{Urine}

For the urine-QC, mean inter-assay CV was $11.4 \%$, whilst the intra-assay CV was $4.9 \%$. A low epicatechin urine sample was used as the matrix into which epicatechin was spiked in order to produce a calibration curve ranging from 0 $1000 \mathrm{ng} / \mathrm{ml}$. Two urine samples per batch were randomly selected and spiked with $200 \mathrm{ng} / \mathrm{ml}$ of epicatechin to assess recovery $($ recovery $=115.9 \%)$. 